\title{
Decreased bone mineral density in young male veterans on Pioglitazone*
}

\author{
Subhashini Yaturu ${ }^{1 \#}$, Jared Davis ${ }^{2}$, Runhua Shi $^{3}$ \\ ${ }^{1}$ Section of Endocrinology and Metabolism, Stratton VA Medical Center, New York, USA; \\ ${ }^{\#}$ Corresponding Author: Subhashini.Yaturu@va.gov; yaturu@yahoo.com \\ ${ }^{2}$ Overton Brooks VA Medical Center, East Stoner, Shreveport, USA; Jared.Davis@va.gov \\ ${ }^{3}$ Fiest-Weiler Cancer Center, Louisiana State University Health Sciences Center (LSUHSC), Shreveport, USA; RShi@1suhsc.edu
}

Received 7 December 2011; revised 12 January 2012; accepted 27 January 2012

\section{ABSTRACT}

Background and Objective: Epidemiological and observational studies indicate that thiazolidinedione (TZD) therapy with rosiglitazone and Pioglitazone is associated with an increased risk of fractures. The effect of TZDs on bone mineral density (BMD) in men with type 2 diabetes is still in debate. The objective of the study was to investigate changes in BMD and bone turnover markers (BTM) associated with Pioglitazone use in men. Design and Methods: This prospective cross sectional comparative study evaluated the changes in BMD and BTM in male veterans aged less than 55 years, with diabetes with or without use of Pioglitazone. In a 6 month follow up study, main outcome measures included BMD at AP spine, femur and wrist; and BTM (osteocalcin and $(\mathrm{CTx}$ ) at a referral center, with no interventions. Results: Pioglitazone use was associated with significant decrease in BMD (annualized \% change of $>3 \%$ ) at femoral neck, total hip and $1 / 3^{\text {rd }}$ radius; increase in CTx by $29 \%$ and decrease in osteocalin by $20 \%$ at 6 months. Conclusions: Even in young men Pioglitazone use was associated with bone loss. The changes in BTM suggest effect of Pioglitazone on both osteoblast and osteoclast activity.

Keywords: Pioglitazone; Bone Mineral Density; Bone Markers; DXA; Type 2 Diabetes; Men

\section{INTRODUCTION}

Osteoporosis and type 2 diabetes (DM), two of the most common chronic disease conditions, represent ma-

\footnotetext{
*Disclosures: Dr. Yaturu and Jared Davis received the salary support from VA Medical Center. Dr. Yaturu Received VA Merit Review Grant for the study.

Clinical Trials registration: NCT00467285.
}

jor public health burdens. Subjects with DM have a higher risk of hip fractures [1-6] as well as fractures at other sites $[5,7,8]$. Hip fractures are associated with significant morbidity and mortality and result in increased health care costs [9]. Thiazolidinediones (TZDs) are an important class of insulin-sensitizing antidiabetic agents, with a plethora of beneficial effects in addition to improving hyperglycemia. The pleiotropic effects of TZDs include decreasing insulin resistance, hyperplasia of pancreatic beta cells (potential to improve beta cell function), improve the lipid parameters, alteration in adipokines (increase the adiponectin, decrease resistin), decreasing C-reactive protein levels, improving endothelial function, reducing restenosis rates in subjects with coronary artery disease, mild improvement in blood pressure and decreasing urinary albumin excretion. However, epidemiological and observational studies indicate that TZD therapy with rosiglitazone and Pioglitazone is also associated with an increased risk of fractures [10-15]. TZDs improve glycemic control through activation of the peroxisome proliferator-activated receptor (PPAR)- $\gamma$. TZDs inhibit osteoblastogenesis [16-18] and suppress markers of osteoblast activity [16], and cause increased osteoclastogenesis [19], which results in decreased bone mass. A short-term study in premenopausal women with polycystic ovary syndrome (PCOS) reported decreased BMD with an average decline of $1.1 \%$ at lumbar spine and $1.4 \%$ at femoral neck after 16 weeks of treatment, as well as a significant decrease in alkaline phosphatase but no changes in osteocalcin [20]. Studies in women with TZDs have shown decreased BMD [21]. Very limited data exists concerning the effects of TZDs on BMD in men. The aim of this study was to evaluate the effects of the TZD Pioglitazone on BMD and bone turnover markers in young men with type 2 diabetes.

\section{MATERIALS AND METHODS}

This is a single-center open-label prospective clinical study. The study was carried out at Overton Brooks VA 
Medical Center at Shreveport, Louisiana after the approval of the protocol and consent form Institutional Review Board at LSUHSC, Shreveport as well as the Research and Development Committee at VAMC. All the subjects have provided informed consent. The inclusion criteria included men aged 30 - 55 years of all ethnic groups with type 2 diabetes. There were 92 participants with type 2 diabetes, with a mean age of 48 years. Among them, 28 subjects were being treated with pioglitazone; the control group comprised 64 subjects with diabetes matched for age, ethnicity, body mass index (BMI) and smoking and alcohol history that were not being treated with Pioglitazone or rosiglitazone. To avoid the confounding factors of possible unequal Vitamin D and calcium intake, all subjects were given vitamin D and calcium supplements (400 Units Vitamin D with calcium $600 \mathrm{mg}$ tab one twice daily).

Exclusion criteria included subjects with increased bone turnover such as immobilization, chronic renal insufficiency even if creatinine is normal (subjects with decreased glomerular filtration rate (GFR)), chronic steroid use, primary hyperparathyroidism, untreated subclinical or clinical hyperthyroidism, or Paget's disease; Vitamin D deficiency $(<20 \mathrm{mg} \%)$, subjects with known histories of chronic pancreatitis, pancreatectomy or malabsorption syndromes; diseases associated with altered bone mineral metabolism: untreated hyperthyroidism, Cushing's syndrome, hyperparathyroidism, chronic kidney disease; or those subjects taking medications that alter bone mineral metabolism. These include glucocorticoids, bisphosphonates, calcitonin, anticonvulsants, immunosuppressants, gonadal hormones (testosterone or estrogens) and gonadotropin-releasing hormones. Female subjects were excluded to avoid perimenopausal and postmenopausal effects on bone turnover within that age group. We measured changes in bone mineral density (BMD) and bone turnover markers in subjects who had just begun taking Pioglitazone, and in a control group of diabetic subjects not receiving Pioglitazone, at baseline and at follow up at 6 and 12 months later. The study involved three visits: one at baseline, then one each at 6 and 12 months after the initial visit. The primary outcome measure was to determine changes in BMD and the secondary end points included changes in bone turnover markers (BTM). BTM includes osteocalcin and bone specific alkaline phosphatase as markers of osteoblast activity, and serum C-telopeptide (CTx) and urinary Ntelopeptide as markers of osteoclast activity.

\subsection{BMD Measurements}

BMD was measured using dual energy x-ray absorptiometry (DXA), using Lunar Prodigy at the AP and lateral lumbar spine (L1-4), at the proximal femur (total hip and femoral neck), and at 33\% radius. DXA quality assurance measurements were performed to ensure scanner reliability. The precision of DXA scans is $1 \%-1.8 \%$. Body composition analysis, including the percentage of body fat and total BMD, was measured at the time of BMD measurement.

\subsubsection{Laboratory Measurements}

Bone specific alkaline phosphatase (BAP) assays were carried out using an EI kit from Immunodiagnostics Systems Inc. (Ostase ${ }^{\circledR}$ assay) with a sensitivity of $<1 \mu \mathrm{g} / \mathrm{L}$ and a specificity of $100 \%$, and intra- and interassay variability of $<6 \%$. Assays for 1,25-dihydroxyvitamin D and 25-hydroxyvitamin $\mathrm{D}$ were carried out using enzyme immunoassay (EI) kits from Immunodiagnostics Systems Inc., with sensitivities of $2.5 \mathrm{pg} / \mathrm{mL}$ and $5 \mathrm{nmol} / \mathrm{L}$, respectively. Both the assays have a specificity of $100 \%$. The intra- and interassay variability of the 1,25-dihydroxyvitamin $\mathrm{D}$ and 25-Hydroxyvitamin $\mathrm{D}$ assays were $6 \%-7 \%$ and $<7 \%$, respectively. Osteocalcin was measured using an Osteocalcin (Intact) ELISA from Alpco with a sensitivity of $0.4 \mathrm{ng} / \mathrm{mL}$ and an intra- and interassay variability of $5 \%-7 \%$.

\subsubsection{Statistical Analysis}

Statistics were performed in Microsoft Excel 2007. All data are presented as means \pm S.D. Pretreatment differences between patients with diabetes in the pioglitazone and non-Pioglitazone groups were tested using unpaired $t$ tests. The effects of Pioglitazone and placebo treatment were evaluated by performing paired $t$ tests. A $p$ value of $<0.05$ was considered significant.

\section{RESULTS}

Baseline characteristics of the cohort for both control and Pioglitazone subgroups are shown in Table 1. The demographic and clinical characteristics, including age, BMI, duration of DM, glycemic control and blood pressure, were similar among both groups at study entry. Parathyroid hormone levels and $25(\mathrm{OH})$ Vitamin D and 1, $25(\mathrm{OH})$ Vitamin D levels were in the normal range both at baseline and at follow up.

\subsection{BMD and Bone Metabolic Parameters}

The BMD data at baseline in both groups is shown in Table 2. Changes in BMD at 6months follow up (represented as annualized \% change) is shown in Figure 1. As the study is terminated before completion only limited subjects had follow up at 12 months. There was continued decrease in BMD at 12 months.

\subsubsection{Change in BMD at Lumbar Spine}

There were no significant changes in BMD in either 
Table 1. Shows the baseline clinical parameters in both groups.

\begin{tabular}{cccc}
\hline & Pioglitazone & No Pioglitazone & $p$-Value \\
\hline Age in years & $49 \pm 6$ & $49 \pm 3$ & NS \\
BMI & $33.6 \pm 5$ & $35.2 \pm 5$ & NS \\
Systolic BP & $131 \pm 14$ & $129 \pm 17$ & NS \\
Diastolic BP & $77 \pm 9$ & $78 \pm 10$ & NS \\
HgA1C & $7.54 \pm 1.7$ & $7.45 \pm 2.1$ & NS \\
Glucose (mg/dl) & $155 \pm 80$ & $150 \pm 45$ & NS \\
Creatinine (mg/dl) & $1.01 \pm 0.12$ & $0.99 \pm 0.17$ & NS \\
\hline
\end{tabular}

BMI: Body mass index; BP: blood pressure; NS: not significant.

Table 2. Shows the baseline BMD data.

\begin{tabular}{ccc}
\hline BMD Site & Pioglitazone & No Pioglitazone \\
\hline AP Spine & $1.303 \pm 0.12$ & $1.266 \pm 0.15$ \\
Femoral Neck & $1.095 \pm 0.14$ & $1.055 \pm 0.13$ \\
Total R Hip & $1.156 \pm 0.14$ & $1.117 \pm 0.14$ \\
Radius 33\% & $1.002 \pm 0.08$ & $1.017 \pm 0.09$
\end{tabular}

BMI: Body mass index; BMD: bone mineral density.

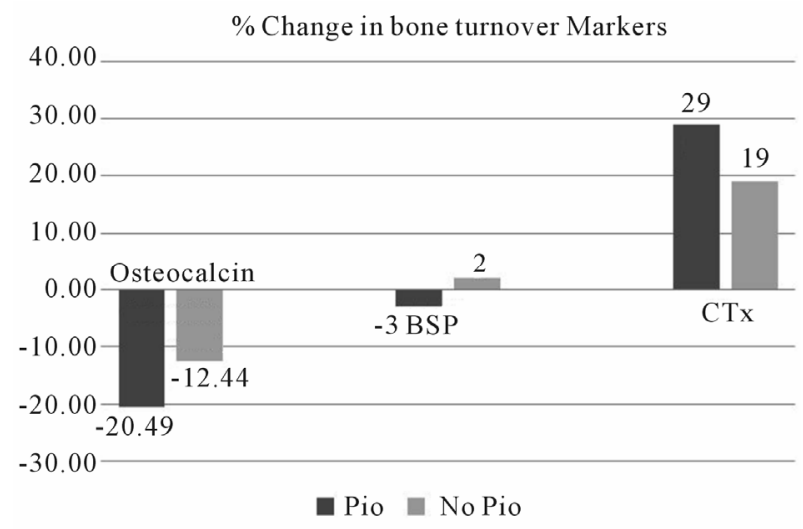

Figure 1. Changes in BMD at 6 months follow up in young men with Pioglitazone use compared to no Pioglitazone use. BMD: bone mineral density; Neck: Femoral neck.

group at follow up compared to baseline or between groups.

\subsubsection{Change in BMD at Hip}

There was a significant decrease in BMD for both total hip and femoral neck in subjects in the Pioglitazone group, as shown in Figure 1. In the control subjects, there was no significant interval change.

\subsubsection{Change in BMD at Wrist ( 0.33 Radius)}

There was a significant decrease in BMD at $1 / 3^{\text {rd }}$ radius in subjects in the Pioglitazone group, as shown in Figure
1. In the control subjects, there was no significant interval change.

\subsection{Bone Turnover Markers}

The bone turnover markers measured include bone specific alkaline phosphatase, osteocalcin and carboxyterminal collagen crosslinks (CTx) in the serum and the urinary N-telopeptide (NTx) /creatinine index. Changes in the levels of osteocalcin, bone specific alkaline phosphatase and CTx are shown in Figure 2.

\section{DISCUSSION}

The subjects included in this study have type 2 diabetes, but are otherwise healthy, which allowed us to assess the effects of Pioglitazone on bone metabolism independent of any confounding factors. In this study, we documented significantly decreased BMD levels during treatment with Pioglitazone in a group of young men with type 2 diabetes. So far, the randomized trials with RSG and PIO have reported on treatment for only 14 - 16 weeks.

There are some interesting features in this study. First, the study involves young men with type 2 diabetes on Pioglitazone. Second, a significant decrease was measured in BMD at distal radius. At 6 months follow up there was an average annualized percentage decrease in BMD of $3.2 \%$ at femoral neck. The study examined changes to bone health in young men with type 2 diabetes without any secondary causes for increased bone turnover. ALP and osteocalcin levels decreased significantly during treatment with Pioglitazone, suggesting decreased osteoblast activity. No significant changes were noted in $25(\mathrm{OH})$ Vitamin D or 1, $25(\mathrm{OH})$ vitamin D levels.

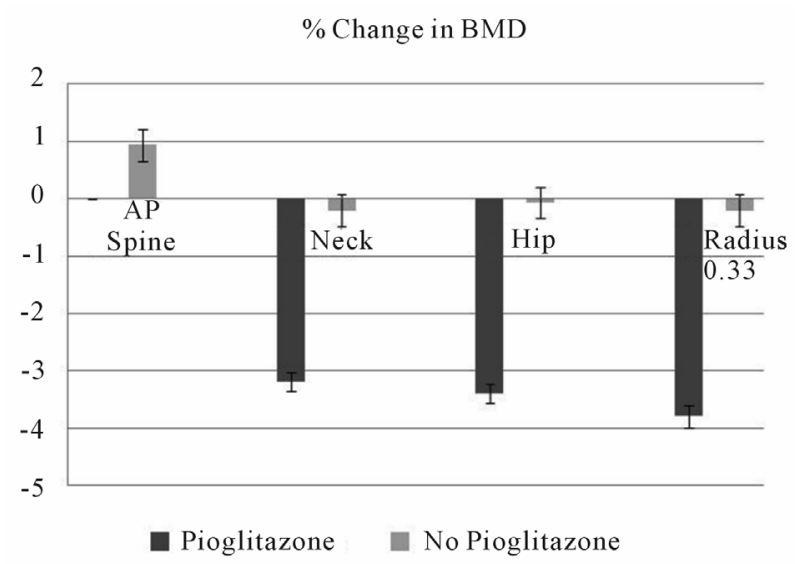

Figure 2. Changes in bone turnover markers in young men with Pioglitazone use compared to no pioglitazoone use. CTx: serum C-telopeptide; BSP: Bone specific alkaline phosphotase; Pio: Pioglitazone. 


\subsection{BMD}

In short-term studies in healthy women, bone loss at the total hip of $1.5 \%-1.7 \%$ was reported over $14-16$ weeks with the use of Pioglitazone in subjects with polycystic ovary syndrome [20] or rosiglitazone in healthy premenopausal women [22]. Schwartz et al. [21] in an observational study, reported loss attributed to TZD use in women as $1.23 \%$ per year at the lumbar spine and less than $1 \%$ at hip, with no increased bone loss observed in diabetic men. In contrast, Yaturu et al. [23] in an observational study of 160 older diabetic men (average age 68 years), reported that RSG use $(N=32)$ was associated with increased bone loss of $1 \%$ to $1.24 \%$ per year at the total hip, femoral neck, and the spine. The differences in the rates of bone loss reported in the literature may reflect an initial period of more rapid bone loss, followed by continued loss at a lower rate, similar to the effects observed with glucocorticoids.

\subsection{Bone Markers}

Our findings are in agreement with those reported in other studies [22,24] concerning the changes in bone turnover markers. In a one short-term study, bone-specific ALP levels were found to be statistically significantly lower 12 weeks after initiation of rosiglitazone treatment [24]. Gray et al. reported significantly reduced serum procollagen type I N-terminal propeptide osteocalcin levels during rosiglitazone treatment, but observed that levels of C-terminal telopeptide (S-CTX) of type I collagen remained stable [22].

In a 16-week randomized placebo-controlled trial among 30 premenopausal women with polycystic ovary syndrome (PCOS), levels of alkaline phosphatase were found to be decreased in the Pioglitazone group compared to those of the placebo group, while levels of osteocalcin and C-telopeptide of type I collagen were not [20]. Based on the markers of bone metabolism, A Diabetes Outcome Progression Trial (ADOPT) suggests that changes in bone resorption as the result of treatment with TZD may be partly responsible for the increased risk of fracture in women taking TZDs [24]. The details of the fracture rates reported in the ADOPT study, which compared treatment with rosiglitazone with that of metformin or glyburide, reveal calculated risk ratios showing that the largest increases in fracture risk were for the foot (RR $=3.3)$, the hand $(\mathrm{RR}=2.6)$, and the proximal humerus $(\mathrm{RR}>8)$. Our data, which document the decreased BMD at the wrist and hip, may explain the increased fracture rates observed in the long bones.

Postmenopausal women have less bone mass to start with, and the rate of bone remodeling is greater, resulting in increased osteoporosis-related fractures. A decrease in BMD has been noted with TZD treatment in both men and women. With regard to TZD use and fractures, the problem exists in both men and women, although the numbers are much higher in postmenopausal women than in those who have not yet gone through menopause. There is a lack of data concerning treatment recommendations for skeletal protection in patients taking TZDs.

Use of bisphosphonates, parathyroid hormone (PTH), or denosumab, the current therapies available for treatment of osteoporosis, have not been tested in relation to TZDs. Given the growing body of evidence linking increased fracture risk and bone loss with TZD use, clinical research is needed to discover treatments that might prevent TZD-induced bone loss.

\section{CONCLUSION}

In conclusion, treatment of young men with type 2 diabetes with Pioglitazone was followed by significantly decreased BMD at the hip, the lumbar spine, and the distal radius. The treated group's sera also showed altered markers of bone mineral turnover, which are surrogates for bone remodeling, implying involvement of both osteoblast and osteoclast activity. Since bone loss is a potent predictor of fracture risk, these results suggest that TZD use may be associated with a considerable burden on skeletal health. Further research is needed to identify the groups that are most susceptible to TZD-induced osteoporosis, to determine the rate of bone loss associated with long-term TZD treatment, and to identify treatments that prevent TZD-induced fracture risk.

\section{AUTHOR CONTRIBUTIONS}

Y.S.: Primary investigator, collection of data, Manuscript writing; JD: Collection of data, carried out assays; RS: Power analysis, statistics.

\section{ACKNOWLEDGEMENTS}

YS was supported by a VA merit review grant and salary support from VHA.

\section{REFERENCES}

[1] Strotmeyer, E.S. and Cauley, J.A. (2007) Diabetes mellitus, bone mineral density, and fracture risk. Current Opinion in Endocrinology, Diabetes and Obesity, 14, 429-435. doi:10.1097/MED.0b013e3282f1cba3

[2] Janghorbani, M., Feskanich, D., Willett, W.C. and Hu, F. (2006) Prospective study of diabetes and risk of hip fracture: The nurses' health study. Diabetes Care, 29, 15731578. doi: $10.2337 / \mathrm{dc} 06-0440$

[3] Strotmeyer, E.S., Cauley, J.A., Schwartz, A.V., Nevitt, M.C., Resnick, H.E., Bauer, D.C., Tylavsky, F.A., de Rekeneire, N., Harris, T.B. and Newman, A.B. (2005) Nontraumatic fracture risk with diabetes mellitus and 
impaired fasting glucose in older white and black adults: The health, aging, and body composition study. Archives of Internal Medicine, 165, 1612-1617. doi:10.1001/archinte.165.14.1612

[4] Cauley, J.A., Lui, L.Y., Ensrud, K.E., Zmuda, J.M., Stone, K.L., Hochberg, M.C. and Cummings, S.R. (2005) Bone mineral density and the risk of incident nonspinal fractures in black and white women. Journal of the American Medical Association (JAMA), 293, 2102-2108. doi:10.1001/jama.293.17.2102

[5] Schwartz, A.V. and Sellmeyer, D.E. (2004) Women, type 2 diabetes, and fracture risk. Current Diabetes Reports, $\mathbf{4}$, 364-369. doi:10.1007/s11892-004-0039-Z

[6] Nicodemus, K.K. and Folsom, A.R. (2001) Type 1 and type 2 diabetes and incident hip fractures in postmenopausal women. Diabetes Care, 24, 1192-1197. doi:10.2337/diacare.24.7.1192

[7] Wallace, C., Reiber, G.E., LeMaster, J., Smith, D.G., Sullivan, K., Hayes, S. and Vath, C. (2002) Incidence of falls, risk factors for falls, and fall-related fractures in individuals with diabetes and a prior foot ulcer. Diabetes Care, 25, 1983-1986. doi:10.2337/diacare.25.11.1983

[8] Schwartz, A.V., Hillier, T.A., Sellmeyer, D.E., Resnick, H.E., Gregg, E., Ensrud, K.E., Schreiner, P.J., Margolis, K.L., Cauley, J.A., Nevitt, M.C., Black, D.M. and Cummings, S.R. (2002) Older women with diabetes have a higher risk of falls: A prospective study. Diabetes Care, 25, 1749-1754. doi:10.2337/diacare.25.10.1749

[9] Ohldin, A. and Floyd, J. (2003) Unrecognized risks among Veterans with hip fractures: Opportunities for improvements. Journal of the Southern Orthopaedic Association, 12, 18-22.

[10] Kahn, S.E., Haffner, S.M., Heise, M.A., Herman, W.H., Holman, R.R., Jones, N.P., Kravitz, B.G., Lachin, J.M., O’Neill, M.C., Zinman, B. and Viberti, G. (2006) Glycemic durability of rosiglitazone, metformin, or glyburide monotherapy. New England Journal of Medicine, 355, 2427-2443. doi:10.1056/NEJMoa066224

[11] Douglas, I.J., Evans, S.J., Pocock, S. and Smeeth, L. (2009) The risk of fractures associated with thiazolidinediones: A self-controlled case-series study. PLoS Medicine, 6, e1000154. doi:10.1371/journal.pmed.1000154

[12] Jones, S.G., Momin, S.R., Good, M.W., Shea, T.K. and Patric, K. (2009) Distal upper and lower limb fractures associated with thiazolidinedione use. American Journal of Managed Care, 15, 491-496.

[13] Dormuth, C.R., Carney, G., Carleton, B., Bassett, K. and Wright, J.M. (2009) Thiazolidinediones and fractures in men and women. Archives of Internal Medicine, 169, 1395-1402. doi:10.1001/archinternmed.2009.214

[14] Bodmer, M., Meier, C., Kraenzlin, M.E. and Meier, C.R. (2009) Risk of fractures with glitazones: A critical review of the evidence to date. Drug Safety, 32, 539-547. doi:10.2165/00002018-200932070-00001

[15] Bilik, D., McEwen, L.N., Brown, M.B., Pomeroy, N.E., Kim, C., Asao, K., Crosson, J.C., Duru, O.K., Ferrara, A., Hsiao, V.C., Karter, A.J., Lee, P.G., Marrero, D.G., Selby, J.V., Subramanian, U. and Herman, W.H. (2010) Thia- zolidinediones and fractures: Evidence from translating research into action for diabetes. Journal of Clinical Endocrinology \& Metabolism, 95, 4560.

[16] Lecka-Czernik, B., Gubrij, I., Moerman, E.J., Kajkenova, O., Lipschitz, D.A., Manolagas, S.C. and Jilka, R.L. (1999) Inhibition of Osf2/Cbfa1 expression and terminal osteoblast differentiation by PPARgamma2. Journal of Cellular Biochemistry, 74, 357-371.

doi:10.1002/(SICI)1097-4644(19990901)74:3<357::AIDJCB5>3.0.CO;2-7

[17] Lecka-Czernik, B., Moerman, E.J., Grant, D.F., Lehmann, J.M., Manolagas, S.C. and Jilka, R.L. (2002) Divergent effects of selective peroxisome proliferator-activated receptor-gamma 2 ligands on adipocyte versus osteoblast differentiation. Endocrinology, 143, 2376-2384. doi:10.1210/en.143.6.2376

[18] Gimble, J.M., Robinson, C.E., Wu, X., Kelly, K.A., Rodriguez, B.R., Kliewer, S.A., Lehmann, J.M. and Morris, D.C. (1996) Peroxisome proliferator-activated receptorgamma activation by thiazolidinediones induces adipogenesis in bone marrow stromal cells. Molecular Pharmacology, 50, 1087-1094.

[19] Ali, A.A., Weinstein, R.S., Stewart, S.A., Parfitt, A.M., Manolagas, S.C. and Jilka, R.L. (2005) Rosiglitazone causes bone loss in mice by suppressing osteoblast differentiation and bone formation. Endocrinology, 146, 1226-1235. doi:10.1210/en.2004-0735

[20] Glintborg, D., Andersen, M., Hagen, C., Heickendorff, L. and Hermann, A.P. (2008) Association of Pioglitazone treatment with decreased bone mineral density in obese premenopausal patients with polycystic ovary syndrome: A randomized, placebo-controlled trial. Journal of Clinical Endocrinology \& Metabolism, 93, 1696-1701. doi:10.1210/jc.2007-2249

[21] Schwartz, A.V., Sellmeyer, D.E., Vittinghoff, E., Palermo, L., Lecka-Czernik, B., Feingold, K.R., Strotmeyer, E.S., Resnick, H.E., Carbone, L., Beamer, B.A., Park, S.W., Lane, N.E., Harris, T.B. and Cummings, S.R. (2006) Thiazolidinedione use and bone loss in older diabetic adults. Journal of Clinical Endocrinology \& Metabolism, 91, 3349-3354. doi:10.1210/jc.2005-2226

[22] Grey, A., Bolland, M., Gamble, G., Wattie, D., Horne, A., Davidson, J. and Reid, I.R. (2007) The peroxisome proliferator-activated receptor-gamma agonist rosiglitazone decreases bone formation and bone mineral density in healthy postmenopausal women: A randomized, controlled trial. Journal of Clinical Endocrinology \& Metabolism, 92, 1305-1310. doi:10.1210/jc.2006-2646

[23] Yaturu, S., Bryant, B. and Jain, S.K. (2007) Thiazolidinedione treatment decreases bone mineral density in type 2 diabetic men. Diabetes Care, 30, 1574-1576. doi:10.2337/dc06-2606

[24] Berberoglu, Z., Gursoy, A., Bayraktar, N., Yazici, A.C., Bascil Tutuncu, N. and Guvener Demirag, N. (2007) Rosiglitazone decreases serum bone-specific alkaline phosphatase activity in postmenopausal diabetic women. Journal of Clinical Endocrinology \& Metabolism, 92, 3523-3530. doi:10.1210/jc.2007-0431 